\title{
A Brief Account of the Relations between Gray-Scale Mathematical Morphologies
}

\author{
Peter Sussner and Marcos Eduardo Valle \\ Institute of Math., Stat. and Sci. Comp. \\ State University of Campinas \\ Campinas, SP 13081-970 \\ E-mail: sussner, mevalle@ime.unicamp.br
}

\begin{abstract}
Mathematical morphology was originally conceived as a set theoretic approach for the processing of binary images. Approaches that extend classical binary morphology to gray-scale images are either based on umbras, thresholds, level sets, or fuzzy sets. Complete lattices form a general framework for all of these approaches. This paper discusses and compares several approaches to gray-scale mathematical morphology including the threshold, umbra, and level set approaches as well as fuzzy approaches.
\end{abstract}

\section{Introduction}

In the early 1960's, Matheron and Serra invented mathematical morphology as a part of binary image processing that is concerned with image filtering and geometric analysis by means of structuring elements [11, 15]. Relying heavily on the early work of Minkowski and Hadwiger on geometric measure theory and integral geometry $[12,8]$, Matheron and Serra succeeded in developing a collection of tools, called morphological operators, that proved to be extremely useful for the analysis of shape and structure in binary images.

The most general mathematical framework in which mathematical morphology can be conducted is given by complete lattices $[16,9,5]$. Ronse formulates necessary and sufficient conditions for gray-scale dilation and erosion [14]. These conditions reveal that every dilation, erosion respectively, is associated with a structuring element. Unlike Ronse, Banon focusses on translation-invariant operators, which play a central role in mathematical morphology, and provides several characterizations of translationinvariant dilations and erosions [3, 4].

Traditionally, binary images are represented as subsets of $\mathbb{R}^{n}$ while gray-scale images are represented as functions
$\mathbb{R}^{n} \rightarrow \mathbb{G}$, where $\mathbb{G} \subseteq \overline{\mathbb{R}}=\mathbb{R} \cup \infty \cup-\infty$ represents a certain set of gray values. Serra was the first to extend binary mathematical morphology to gray-scale images using the threshold approach [15]. An application of a morphological operator to a gray-scale image a results in another grayscale image $\mathbf{b}$ such that $\mathbf{b}(\mathbf{x})$ is determined by the function values of $\mathbf{a}$ in a neighborhood of $\mathbf{x}$.

The most widely known method for the generalization of binary mathematical morphology to gray-scale image processing employs the notion of umbra and is due to Sternberg [18]. The umbra of a gray-scale image $\mathbf{a}: \mathbb{R}^{n} \rightarrow \mathbb{G}$ is given by a subset $\mathcal{U}$ of $\mathbb{R}^{n+1}$ which can be transformed using the tools of binary morphology.

Another approach to gray-scale morphology consists of thresholding an image at all levels and applying binary morphological operators at each of these levels [9]. The resulting sets are combined to synthesize the transformed image.

The two basic morphological operators are erosion and dilation. Erosion marks structuring element origin locations at which a structuring element fits within an image. This concept can be expressed in terms of set inclusion or subsethood. Approaches to gray-scale mathematical morphology can be derived from fuzzy set theory by fuzzyfing the notion of set inclusion. Depending on the particular choice of fuzzy set inclusion [20,10,2], we obtain different notions of fuzzy erosion provided that the operation of fuzzy set inclusion commutes in the second argument with the infimum operator. Examples include the approaches of De Baets [1], Bloch and Maître [6] as well as Sinha and Dougherty [17].

Fuzzy dilation is usually defined as the dual of fuzzy erosion. The notion of duality that is used varies among the researchers of fuzzy mathematical morphology. Many researchers - including Bloch and Maître, Kitainik, and Sinha and Dougherty - introduce a duality relation based on some concept of negation [6, 10, 17]. Other researchers such as Deng and Heijmans advocate a duality relation based on the notion of adjunction [7]. 
The paper is organized as follows. First, we review the basic concepts of mathematical morphology whose mathematical foundations lie in lattice theory and whose origins can be found in the processing of binary images. Section 3 discusses traditional approaches to gray-scale mathematical morphology and establishes connections between these approaches. After providing some background information on fuzzy set theory, we show that most approaches to fuzzy mathematical morphology are based on fuzzy inclusion and intersection measures of a certain form that we call fuzzy Inf-I inclusion and Sup-C Intersection measures. Finally, we relate these fuzzy mathematical morphologies to the traditional approaches towards gray-scale mathematical morphology that we discussed in Section 3.

\section{A Brief Review of the Basic Concepts of Mathematical Morphology}

\subsection{The Complete Lattice Framework for Mathe- matical Morphology}

The mathematical foundations of morphology can be found in lattice theory which is concerned with algebraic structure that arise by imposing some type of ordering on a set $[5,9,14]$.

For example, a binary relation $\leq$ on a set $X \neq \emptyset$ is called a partial ordering if and only if $\leq$ satisfies the following properties for all $x, y, z \in X: 1$ - Reflexivity: $x \leq x, 2$ Anti-Symmetry: $x \leq y, y \leq x \Rightarrow x=y$, and 3 - Transitivity: $x \leq y, y \leq z \Rightarrow x \leq z$;

A set $X$ that is equipped with a partial ordering is called partially ordered set or simply poset. Let $X$ be partially ordered and let $Y \subseteq X$. An element $l \in X$ is called lower bound of $Y$ if $l \leq y$ for all $y \in Y$. Note that $l$ does not necessarily belong to $X$. The notion of upper bound is defined in a similar fashion. We say that $l_{0} \in X$ is the greatest lower bound or infimum of $Y$ if and only if $l_{0}$ represents a lower bound of $Y$ and $l \leq l_{0}$ for all other lower bounds $l$ of $Y$. Similarly, we define the notion of least upper bound or supremum. The infimum of $Y$ is denoted by the symbol $\wedge Y$. Alternatively, we write $\bigwedge_{j \in J} y_{j}$ instead of $\wedge Y$ if $Y=\left\{y_{j}: j \in J\right\}$ for some index set $J$. Similar notations are used to denote the supremum of $Y$.

A poset $X$ is called a lattice if and only if there exists an infimum and a supremum for every finite subset of $X$. Suppose that $X$ and $Y$ are lattices.

We speak of a complete lattice $X$ if every (finite or infinite) subset has an infimum and a supremum in $X$. From now on, we will denote a complete lattice by $\mathbb{L}$. Note that the power set of an arbitrary set together with the partial ordering given by " $\subseteq$ " forms a complete lattice. We will conduct binary mathematical morphology on the complete lat- tice $\mathcal{P}(\mathbf{X})$ where the symbol $\mathbf{X}$ denotes the euclidian space $\mathbb{R}^{d}$ or the digital space $\mathbb{Z}^{d}$ throughout the paper.

In gray-scale morphology, we apply the concepts of lattice theory to images, in other words functions from some point set to a set of gray-levels that forms a complete lattice. In this paper, we restrict our attention to images $\mathbf{X} \rightarrow \mathbb{G}$ where the symbol $\mathbb{G}$ stands either for the set of extended real numbers $\overline{\mathbb{R}}=\mathbb{R} \cup\{+\infty,-\infty\}$ or for the set of extended integers $\overline{\mathbb{Z}}=\mathbb{Z} \cup\{+\infty,-\infty\}$ or for the interval $[0,1]$. We denote the set of images $\mathbf{X} \rightarrow \mathbb{G}$ using the symbol $\mathbb{G}^{\mathbf{X}}$. This set inherits the complete lattice structure from $\mathbb{G}$ if we define $\mathbf{a} \leq \mathbf{b}$ for $\mathbf{a}, \mathbf{b} \in \mathbb{G}^{\mathbf{X}}$ as follows:

$$
\mathbf{a} \leq \mathbf{b} \Leftrightarrow \mathbf{a}(\mathbf{x}) \leq \mathbf{b}(\mathbf{x}) \forall \mathbf{x} \in \mathbf{X} .
$$

The basic operations of mathematical morphology are erosion and dilation. In the general complete lattice setting, an erosion is an operator $\varepsilon: \mathbb{L} \rightarrow \mathbb{L}$ that commutes with the infimum operation. In other words, the operator $\varepsilon$ represents an erosion if and only if the following equality holds for every subset $Y \subseteq \mathbb{L}$ :

$$
\varepsilon(\bigwedge Y)=\bigwedge_{y \in Y} \varepsilon(y) .
$$

Similarly, an operator $\delta: \mathbb{L} \rightarrow \mathbb{L}$ that commutes with the supremum operation is called a dilation. In other words, the operator $\delta$ represents a dilation if and only if the following equality holds for every subset $Y \subseteq \mathbb{L}$ :

$$
\delta(\bigvee Y)=\bigvee_{y \in Y} \delta(y) .
$$

The operators of erosion and dilation are often linked in terms of a relationship of duality. Some authors such as Deng and Heijmans [7] advocate the relationship of adjunction since - among other advantages - the compositions of dilations and erosions yield openings and closings if the pairing between erosion and dilation forms an adjunction.

Consider two arbitrary operators $\varepsilon, \delta: \mathbb{L} \rightarrow \mathbb{L}$. We say that $(\varepsilon, \delta)$ is an adjunction on $(\mathbb{L}, \leq)$ if we have

$$
\delta(\mathbf{a}) \leq \mathbf{b} \Leftrightarrow \mathbf{a} \leq \varepsilon(\mathbf{b}) \forall \mathbf{a}, \mathbf{b} \in \mathbb{L} .
$$

The following proposition shows that adjunction constitutes a duality between erosions and dilations since they form a bijection which reverses the order relation in the complete lattice [9].

Proposition 1. Let $\mathbb{L}$ be a complete lattice and consider two operations $\delta, \varepsilon: \mathbb{L} \times \mathbb{L} \rightarrow \mathbb{L}$.

1. If $(\varepsilon, \delta)$ is an adjunction, then $\delta$ is a dilation and $\varepsilon$ is an erosion.

2. For every dilation $\delta$, there is a unique erosion $\varepsilon$ such that $(\varepsilon, \delta)$ is an adjunction. and for every erosion $\varepsilon$, there is a unique dilation $\delta$ such that $(\varepsilon, \delta)$ is an adjunction. 


\subsection{Binary Mathematical Morphology}

Mathematical morphology was initially developed for the analysis of binary images. We identify a binary image with a subset of an Euclidian space or digital space $\mathbf{X}$ $[11,15]$.

Consider subsets $\mathbf{A}, \mathbf{S}$ of $\mathbf{X}$. The binary erosion $D_{\mathcal{B}}(\mathbf{A}, \mathbf{S})$ and the binary dilation $E_{\mathcal{B}}(\mathbf{A}, \mathbf{S})$ are defined in terms of translations of sets. For example, the translation $\mathbf{S}_{\mathbf{x}}$ of $\mathbf{S}$ by $\mathbf{x} \in \mathbf{X}$ is given by $\mathbf{S}_{\mathbf{x}}=\{\mathbf{s}+\mathbf{x}: \mathbf{s} \in S\}$. We have

$$
\begin{gathered}
E_{\mathcal{B}}(\mathbf{A}, \mathbf{S})=\left\{\mathbf{x} \in \mathbf{X}: \mathbf{S}_{\mathbf{x}} \subseteq \mathbf{A}\right\}=\bigcap_{\mathbf{s} \in \check{\mathbf{S}}} \mathbf{A}_{\mathbf{s}} \\
D_{\mathcal{B}}(\mathbf{A}, \mathbf{S})=\left\{\mathbf{x} \in \mathbf{X}: \check{\mathbf{S}}_{\mathbf{x}} \cap \mathbf{A} \neq \emptyset\right\}=\bigcup_{\mathbf{s} \in \mathbf{S}} \mathbf{A}_{\mathbf{s}}=\bigcup_{\mathbf{a} \in \mathbf{A}} S_{\mathbf{a}}
\end{gathered}
$$

Here, the symbol $\check{\mathbf{S}}$ denotes the reflection of $\mathbf{S}$ around the origin. Formally, we have $\check{\mathbf{S}}=\{-\mathbf{s}: \mathbf{s} \in \mathbf{S}\}$. Similarly, $\check{\mathbf{S}}_{\mathbf{x}}$ denotes the reflection of $\mathbf{S}_{\mathbf{x}}$ around the origin. The set $\mathbf{S}$ is called structuring element in the morphological literature.

Note that the definition of erosion in Equation 5 corresponds to Minkowski subtraction and that the definition of dilation in Equation 6 corresponds to Minkowski addition $[12,8]$. Serra [15] slightly diverges from these definitions which are due to Sternberg [19] by defining the dilation of $\mathbf{A}$ by the structuring element $\mathbf{S}$ as $\left\{\mathbf{x} \in \mathbf{X}: \mathbf{S}_{\mathbf{x}} \cap \mathbf{A} \neq\right.$ $\emptyset\}=D_{\mathcal{B}}(\mathbf{A}, \breve{\mathbf{S}})$.

Theorem 1. Binary dilation is the dual of binary erosion with respect to the relationship

$$
D_{\mathcal{B}}(\mathbf{A}, \mathbf{S})=E_{\mathcal{B}}\left(\mathbf{A}^{c}, \check{\mathbf{S}}\right)^{c} .
$$

Here, $\mathbf{A}^{c}$ denotes the complement of $\mathbf{A}$. The binary dilation $D_{\mathcal{B}}(., \mathbf{S})$ by an arbitrary, fixed structuring element $\mathbf{S}$ and the binary erosion $E(., \mathbf{S})$ by $\mathbf{S}$ also represent adjoint operators in the sense of Equation 4:

$$
D_{\mathcal{B}}(\mathbf{A}, \mathbf{S}) \subseteq \mathbf{B} \Leftrightarrow \mathbf{A} \subseteq E_{\mathcal{B}}(\mathbf{B}, \mathbf{S}) .
$$

\section{Traditional Approaches towards Gray- Scale Mathematical Morphology}

The tools of binary mathematical morphology are limited and cannot be applied to gray-scale images. Serra and Sternberg have developed successful approaches to extend binary mathematical morphology to gray-scale mathematical morphology in the 1980's [15, 18]. We will refer to these approaches as the threshold and the umbra approach. This paper also includes another approach called the level-sets approach that can be viewed as an extension of the threshold approach [9]. In this section, we are dealing with graylevel images $\mathbf{X} \rightarrow \mathbb{G}$ where $\mathbf{X}$ denotes $\mathbb{R}^{d}$ or $\mathbb{Z}^{d}$ and where $\mathbb{G}$ denotes $\overline{\mathbb{R}}$ or $\overline{\mathbb{Z}}$.

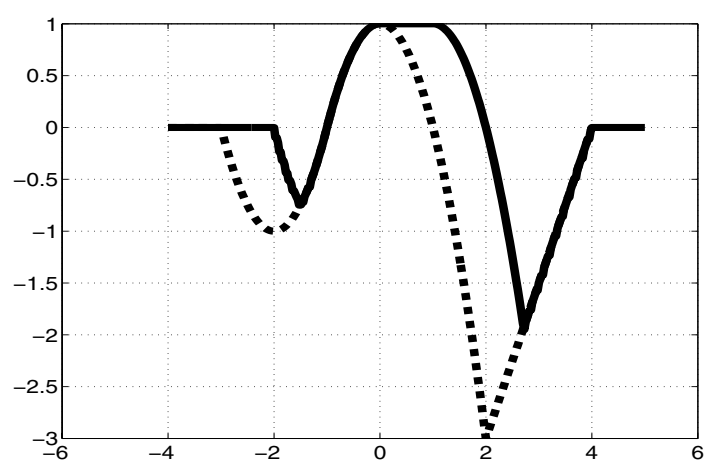

Figure 1. The dashed line refers to $\mathrm{a}$. The continuous line corresponds to $D_{\mathcal{T}}(\mathbf{a}, \mathbf{S})$ where $\mathbf{S}=[0,1]$.

\subsection{The Threshold Approach or Flat Approach}

The threshold approach dates back to Serra's work [15]. Given a gray-scale image $\mathbf{a}$ and a structuring element $\mathbf{S} \subseteq$ $\mathbf{X}$, we define the $T$-dilation $D_{\mathcal{T}}(\mathbf{a}, \mathbf{S})$ and the $T$-erosion $E_{\mathcal{T}}(\mathbf{a}, \mathbf{S})$ [13], also called flat dilation and flat erosion [9], as follows:

$$
\begin{aligned}
& D_{\mathcal{T}}(\mathbf{a}, \mathbf{S})(\mathbf{x})=\bigvee_{\mathbf{y} \in \check{\mathbf{S}}_{\mathbf{x}}} \mathbf{a}(\mathbf{y}), \\
& E_{\mathcal{T}}(\mathbf{a}, \mathbf{S})(\mathbf{x})=\bigwedge_{\mathbf{y} \in \mathbf{S}_{\mathbf{x}}} \mathbf{a}(\mathbf{y}) .
\end{aligned}
$$

Example 1. Figure 1 displays the graph of a gray-scale image $\mathbf{a}: \mathbb{R} \rightarrow \overline{\mathbb{R}}$ as a dashed line. Note that $\mathbf{a}(\mathbf{x})=-\infty$ for all $x \notin[-4,4]$. The threshold dilation $D_{\mathcal{T}}(\mathbf{a}, \mathbf{S})$, where $\mathbf{S}=\{x \in \mathbb{R}: 0 \leq x \leq 1\}$, is shown as a continuous line.

\subsection{The Level Set Approach}

An image $\mathbf{a} \in \mathbb{G}^{\mathbf{X}}$ can be decomposed into its threshold or level sets $\mathcal{S}_{t}(\mathbf{a})$ given by [9]:

$$
\mathcal{S}_{t}(\mathbf{a})=\{\mathbf{x} \in \mathbf{X}: \mathbf{a}(\mathbf{x}) \geq t\} .
$$

The image a can be recovered from its level sets as follows:

$$
\mathbf{a}(\mathbf{x})=\bigvee\left\{t \in \mathbb{G}: \mathbf{x} \in \mathcal{S}_{t}(\mathbf{a})\right\} .
$$

The Equations 11 and 12 yield tools to building operators on the lattice $\mathbb{G}^{\mathbf{X}}$ from operators on $\mathcal{P}(\mathbf{X})$. For a family of set operators $\left\{\psi_{t}: t \in \mathbb{G}\right\}$, we define the operator $\Psi$ : $\mathbb{G}^{\mathbf{X}} \rightarrow \mathcal{P}(\mathbf{X})^{\mathbb{G}}$ as follows:

$$
\Psi(\mathbf{a})(\mathbf{x})=\bigvee\left\{t \in \mathbb{G}: \mathbf{x} \in \psi_{t}\left(\mathcal{S}_{t}(\mathbf{a})\right)\right\} .
$$

The operator $\Psi$ is called a semi-flat operator generated by the family $\left\{\psi_{t}: t \in \mathbb{G}\right\}$ [9]. 
Theorem 2. The following definitions derived from Equation 13 yield a dilation (Equation 14) and an erosion (Equation 15) for fixed $\mathbf{s} \in \mathbb{G}^{\mathbf{X}}$ :

$$
\begin{aligned}
D_{\mathcal{L}}(\mathbf{a}, \mathbf{s})(\mathbf{x}) & =\bigvee\left\{t \in \mathbb{G}: \mathbf{x} \in D_{\mathcal{B}}\left(\mathcal{S}_{t}(\mathbf{a}), \mathcal{S}_{t}(\mathbf{s})\right)\right\} \\
E_{\mathcal{L}}(\mathbf{a}, \mathbf{s})(\mathbf{x}) & =\bigvee\left\{t \in \mathbb{G}: \mathbf{x} \in E_{\mathcal{B}}\left(\mathcal{S}_{t}(\mathbf{a}), \mathcal{S}_{t}(\mathbf{s})\right)\right\}
\end{aligned}
$$

The proof of this theorem makes use of the fact that the operator $D_{\mathcal{L}}(., \mathbf{s}), E_{\mathcal{L}}(., \mathbf{s})$ respectively, represents a semiflat operator that is generated by a family of dilations, a family of erosions respectively [9]. We will speak of the $L$-dilation $D_{\mathcal{L}}(\mathbf{a}, \mathbf{s})$ and of the $L$-erosion $E_{\mathcal{L}}(\mathbf{a}, \mathbf{s})$. The following theorems can be proved by means of straightforward verification. Due to space constraints, detailed proofs cannot be provided in this paper.

Theorem 3. For an arbitrary binary structuring element $\mathbf{S} \subseteq \mathbf{X}$ and for arbitrary $\mathbf{a} \in \mathbb{G}^{\mathbf{X}}$, we have $D_{\mathcal{T}}(\mathbf{a}, \mathbf{S})=$ $D_{\mathcal{L}}(\mathbf{a}, \mathbf{s})$ and $E_{\mathcal{T}}(\mathbf{a}, \mathbf{S})=E_{\mathcal{L}}(\mathbf{a}, \mathbf{s})$ where $\mathbf{s} \in \mathbb{G}^{\mathbf{X}}$ is defined as follows:

$$
\mathbf{s}(\mathbf{x})=\left\{\begin{array}{cc}
\infty & \forall \mathbf{x} \in \mathbf{S} \\
-\infty & \forall \mathbf{x} \notin \mathbf{S}
\end{array}\right.
$$

Example 2. Let a : $\mathbb{R} \rightarrow \overline{\mathbb{R}}$ be the gray-scale image depicted in Figure 1 and let $\mathbf{s}: \mathbb{R} \rightarrow \overline{\mathbb{R}}$ such that $\mathbf{s}(\mathbf{x})=+\infty$ if $x \in[0,1]$ and $\mathbf{s}(\mathbf{x})=-\infty$ if $x \notin[0,1]$. Figure 1 shows the L-dilation of a by s since $D_{\mathcal{L}}(\mathbf{a}, \mathbf{s})=D_{\mathcal{T}}(\mathbf{a}, \mathbf{S})$, where $S=[0,1]$.

Theorem 4. For an arbitrary, but fixed structuring element $\mathbf{s} \in \mathbb{G}^{\mathbf{X}}$, the L-dilation and the L-erosion form an adjunction. Furthermore, for every $\mathbf{a}, \mathbf{b} \in \mathbb{G}^{\mathbf{X}}$ and every $\mathbf{s} \in \mathbb{G}^{\mathbf{X}}$, the L-dilation and the L-erosion are given by

$$
\begin{array}{r}
D_{\mathcal{L}}(\mathbf{a}, \mathbf{s})(\mathbf{x})=\bigvee_{\mathbf{y} \in \mathbf{X}} \mathbf{s}(\mathbf{x}-\mathbf{y}) \wedge \mathbf{a}(\mathbf{y}), \\
E_{\mathcal{L}}(\mathbf{a}, \mathbf{s})(\mathbf{x})=\bigwedge_{\mathbf{y} \in \mathbf{X}} f(\mathbf{s}(\mathbf{y}-\mathbf{x}), \mathbf{a}(\mathbf{y})),
\end{array}
$$

where $f: \mathbb{G} \times \mathbb{G} \rightarrow \mathbb{G}$ is such that

$$
f(x, y)=\left\{\begin{array}{cc}
+\infty, & x \leq y \\
y, & x>y .
\end{array}\right.
$$

Example 3. Let a $: \mathbb{R} \rightarrow \overline{\mathbb{R}}$ be the gray-scale image and let $\mathrm{s}: \mathbb{R} \rightarrow \overline{\mathbb{R}}$ be the structuring element whose graphs are depicted in Figure 2. We would like to clarify that $\mathbf{s}(\mathbf{x})=-\infty$ for all $\mathbf{x} \notin[-1,1]$. The continuous line in Figure 2 represents the L-dilation $D_{\mathcal{L}}(\mathbf{a}, \mathbf{s})$ and the dashed line represents the image $\mathbf{a}$.

\subsection{The Umbra Approach}

In this section, we discuss the umbra approach that represents one of the earliest approaches for extending binary

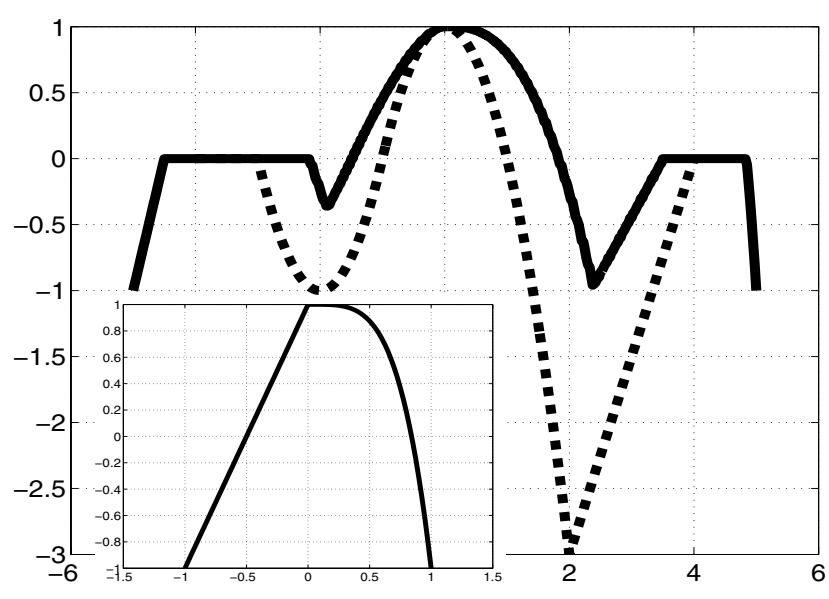

Figure 2. The thin dashed line corresponds to the original image a. The continuous line represents the level set dilation $D_{\mathcal{L}}(\mathbf{a}, \mathbf{s})$ where $\mathbf{s}$ is the structuring element displayed in the bottom left corner.

mathematical morphology to the processing and the analysis of gray-level images [18].

The $U$-dilation $D_{\mathcal{U}}(\mathbf{a}, \mathbf{s})$ and the $U$-erosion $U$-erosion $E_{\mathcal{U}}(\mathbf{a}, \mathbf{s})$ are defined as follows for every $\mathbf{a}, \mathbf{s} \in \mathbb{G}^{\mathbf{X}}[14]$ :

$$
\begin{aligned}
& D_{\mathcal{U}}(\mathbf{a}, \mathbf{s})(\mathbf{x})=\bigvee_{\mathbf{y} \in \mathbf{X}}(\mathbf{a}(\mathbf{y})+\mathbf{s}(\mathbf{x}-\mathbf{y})) \\
& E_{\mathcal{U}}(\mathbf{a}, \mathbf{s})(\mathbf{x})=\bigwedge_{\mathbf{y} \in \mathbf{X}}\left(\mathbf{a}(\mathbf{y})+^{\prime}(-\mathbf{s}(\mathbf{y}-\mathbf{x}))\right) .
\end{aligned}
$$

The operations " + " and " + " " differ with respect to the sum of $\infty$ and $-\infty$ as described in Equations 22 and 23 below. Otherwise, these operations behave as one would expect.

$$
\begin{gathered}
\infty+(-\infty)=(-\infty)+\infty=-\infty \\
\infty+^{\prime}(-\infty)=(-\infty)+^{\prime} \infty=\infty
\end{gathered}
$$

For a given binary structuring element $\mathbf{S} \subseteq \mathbf{X}$, let us construct a gray-scale structuring element $\mathbf{s} \in \mathbb{G}^{\mathbf{X}}$ as follows:

$$
\mathbf{s}(\mathbf{x})=\left\{\begin{array}{cc}
0, & \mathbf{x} \in \mathbf{S}, \\
-\infty, & \mathbf{x} \notin \mathbf{S}
\end{array}\right.
$$

For every image $\mathbf{a} \in \mathbb{G}^{\mathbf{X}}$, we can compute the $\mathrm{U}$ Dilation $D_{\mathcal{U}}(\mathbf{a}, \mathbf{s})(\mathbf{x})$ of a by this structuring element $\mathbf{s}$ as follows

$$
\begin{aligned}
& D_{\mathcal{U}}(\mathbf{a}, \mathbf{s})(\mathbf{x})=\bigvee_{\mathbf{y} \in \mathbf{X}}(\mathbf{a}(\mathbf{x}-\mathbf{y})+\mathbf{s}(\mathbf{y})) \\
= & {\left[\bigvee_{\mathbf{y} \in \mathbf{S}}(\mathbf{a}(\mathbf{x}-\mathbf{y}))\right]=\left[\bigvee_{\mathbf{y} \in \check{\mathbf{S}}_{\mathbf{x}}}(\mathbf{a}(\mathbf{y}))\right] . }
\end{aligned}
$$




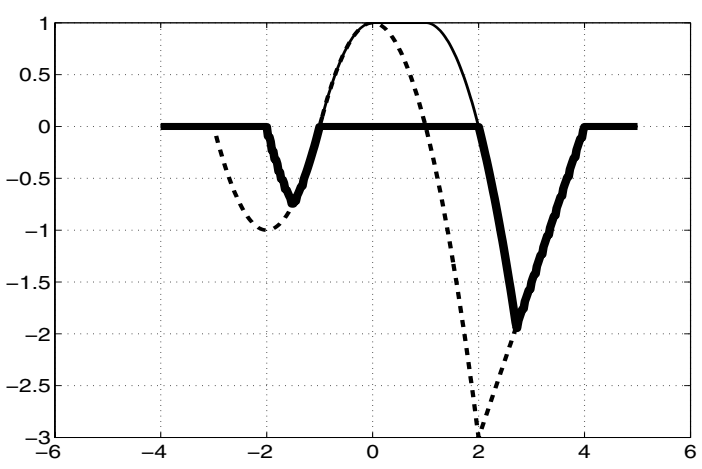

Figure 3. Consider $\mathbf{S}=[0,1]$. Let $\mathbf{s}$ be as in Equation 24 . The original image $\mathrm{a}$ is drawn as a thin dashed line. The thick line and the thin continuos line represent $D_{\mathcal{L}}(\mathbf{a}, \mathbf{s})$ and $D_{\mathcal{T}}(\mathbf{a}, \mathbf{S})$.

In a similar vein, we recognize that

$$
E_{\mathcal{U}}(\mathbf{a}, \mathbf{s})(\mathbf{x})=\bigwedge_{\mathbf{y} \in \mathbf{S}_{\mathbf{x}}} \mathbf{a}(\mathbf{y}) .
$$

In view of the definitions of $T$-dilation and $T$-erosion that we presented in Equations 9 and 10, these observations yield the following theorem.

Theorem 5. For all $\mathbf{a} \in \mathbb{G}^{\mathbf{X}}$ and for all $\mathbf{S} \subseteq \mathbf{X}$, we have the following identities.

$$
D_{\mathcal{T}}(\mathbf{a}, \mathbf{S})=D_{\mathcal{U}}(\mathbf{a}, \mathbf{s}), E(\mathbf{a}, \mathbf{S})=E_{\mathcal{U}}(\mathbf{a}, \mathbf{s}),
$$

where $\mathbf{S}$ is constructed from $\mathbf{S}$ by means of Equation 24.

Example 4. Let $\mathbf{S}$ be the unit interval $[0,1]$. Consider a gray-scale structuring element $\mathbf{s}$ that satisfies Equation 24. Recall that the U-dilation $D_{\mathcal{U}}(\mathbf{a}, \mathbf{s})$ of $\mathbf{a}$ by $\mathbf{s}$ coincides with the T-dilation $D_{\mathcal{T}}(\mathbf{a}, \mathbf{S})$ that is shown in Figure 1 .

Figure 3 compares the L-Dilation $D_{\mathcal{L}}(\mathbf{a}, \mathbf{s})$ and the TDilation $D_{\mathcal{T}}(\mathbf{a}, \mathbf{S})$. The thin dashed line represents the image a. The thick continuous line represents the L-Dilation and the thin dashed line represents the T-Dilation of a by the structuring element $\mathbf{s}$. Note the difference between $D_{\mathcal{L}}(\mathbf{a}, \mathbf{s})$ and $D_{\mathcal{T}}(\mathbf{a}, \mathbf{S})$.

Example 5. Consider the structuring element $\mathbf{s}: \mathbb{R} \rightarrow \overline{\mathbb{R}}$ shown at the bottom of Figure 2. Recall that $\mathbf{s}(\mathbf{x})=-\infty$ for all $\mathbf{x} \notin[-1,1]$. Figure 4 reveals the difference between the U-Dilation $D_{\mathcal{U}}(\mathbf{a}, \mathbf{s})$ - drawn as a thick line - and the L-Dilation $D_{\mathcal{L}}(\mathbf{a}, \mathbf{s})$ - drawn as a thin continuous line.

\section{Fuzzy Mathematical Morphology}

\subsection{Basic Concepts of Fuzzy Set Theory}

Fuzzy set theory extends conventional (crisp) set theory. Lotfi Zadeh introduced this mathematical theory as a tool

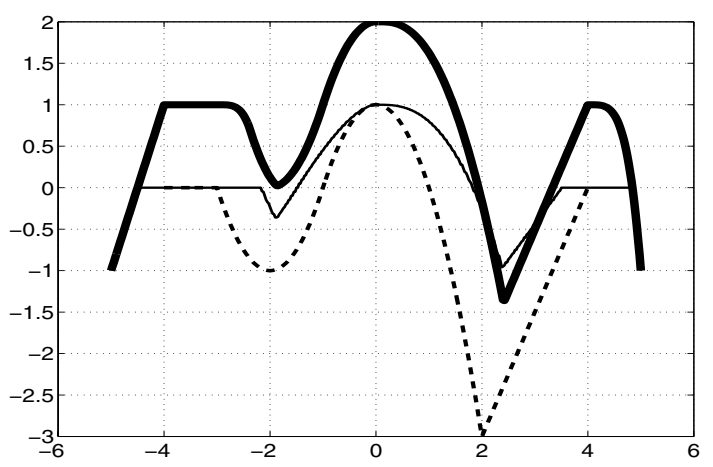

Figure 4. The thick line and the thin continuos line refer to $D_{\mathcal{U}}(\mathbf{a}, \mathbf{s})$ and to $D_{\mathcal{L}}(\mathbf{a}, \mathbf{s})$. The structuring element $\mathbf{s}$ can be found at the bottom of Figure 2 .

to model the vagueness and ambiguity in complex systems [20]. A fuzzy set is formally defined as a function a from a set $\mathbf{X}$ to $[0,1]$. The function a is also called membership function and the value $\mathbf{a}(\mathbf{x})$ is the degree of membership of $\mathbf{x}$ in the fuzzy set $\mathbf{a}$. In particular, we have that $\mathbf{a}(\mathbf{x})=0$ represents complete exclusion and that $\mathbf{a}(\mathbf{x})=1$ represents complete membership. The class of fuzzy sets in $\mathbf{X}$ will be denoted by $\mathcal{F}(\mathbf{X})=[0,1]^{\mathbf{X}}$. Note that fuzzy set theory can be used for the design of image operators since an image $\mathbf{a}: \mathbf{X} \rightarrow[0,1]$ can be interpreted as a fuzzy set of $\mathbf{X}$. From now on, an image $\mathbf{a} \in \mathcal{F}(\mathbf{X})$ will be called fuzzy image. We identify every crisp set $\mathbf{A} \in \mathcal{P}(\mathbf{X})$ with a fuzzy set $\mathbf{a} \in \mathcal{F}(\mathbf{X})$ via the relationship

$$
\mathbf{a}(\mathbf{x})= \begin{cases}1, & \text { if } \mathbf{x} \in \mathbf{A} \\ 0, & \text { else }\end{cases}
$$

We define a fuzzy conjunction as an increasing mapping $C_{\mathcal{F}}:[0,1] \times[0,1] \longrightarrow[0,1]$ that satisfies $C_{\mathcal{F}}(0,0)=$ $C_{\mathcal{F}}(0,1)=C_{\mathcal{F}}(1,0)=0$ and $C_{\mathcal{F}}(1,1)=1$. The minimum operator obviously yields a simple example. Some other particular choices of fuzzy conjunction are due to Lukasiewicz and to Kleene and Dienes:

$$
\begin{aligned}
C_{M}(x, y) & =x \wedge y \\
C_{L}(x, y) & =0 \vee(x+y-1) \\
C_{K D}(x, y) & =\left\{\begin{array}{ll}
0, & y \leq 1-x \\
y, & y>1-x
\end{array} .\right.
\end{aligned}
$$

An operator $I_{\mathcal{F}}:[0,1] \times[0,1] \longrightarrow[0,1]$ that is decreasing in the first argument and that is increasing in the second argument is called a fuzzy implication if $I_{\mathcal{F}}$ extends the usual crisp implication on $\{0,1\} \times\{0,1\}$, i.e. $I_{\mathcal{F}}(0,0)=I_{\mathcal{F}}(0,1)=I_{\mathcal{F}}(1,1)=1$ and $I_{\mathcal{F}}(1,0)=0$. Some particular fuzzy implications, that were introduced by Gödel, Lukasiewicz, and by Kleene and Dienes, can be 
found below.

$$
\begin{aligned}
I_{G}(x, y) & =\left\{\begin{array}{ll}
1, & x \leq y \\
y, & x>y
\end{array},\right. \\
I_{L}(x, y) & =1 \wedge(y-x+1), \\
I_{K D}(x, y) & =(1-x) \vee y .
\end{aligned}
$$

A fuzzy negation is defined as a decreasing operator $N_{\mathcal{F}}$ : $[0,1] \longrightarrow[0,1]$ that satisfies $N_{\mathcal{F}}(0)=1$ and $N_{\mathcal{F}}(1)=0$. A fuzzy negation is called involutive if $N_{\mathcal{F}}\left(N_{\mathcal{F}}(x)\right)=x$ for all $x \in[0,1]$. The following unary operators represent examples of involutive fuzzy negations.

$$
\begin{aligned}
& N_{S}(x)=1-x, \\
& N_{D}(x)=\frac{1-x}{1+p x}, \quad p>-1, \\
& N_{R}(x)=\sqrt[p]{1-x^{p}}, \quad p \in(0, \infty) .
\end{aligned}
$$

We can obtain a fuzzy implication from a fuzzy conjunction and an involutive fuzzy negation as follows:

$$
I_{\mathcal{F}}(x, y)=N_{\mathcal{F}}\left(C_{\mathcal{F}}\left(x, N_{\mathcal{F}}(y)\right)\right), \forall x, y \in[0,1] .
$$

Similarly, a fuzzy conjunction can be derived from a fuzzy implication and an involutive fuzzy negation using the following equation:

$$
C_{\mathcal{F}}(x, y)=N_{\mathcal{F}}\left(I_{\mathcal{F}}\left(x, N_{\mathcal{F}}(y)\right)\right), \forall x, y \in[0,1] .
$$

A fuzzy conjunction $C_{\mathcal{F}}$ is said to be dual to a fuzzy implication $I_{\mathcal{F}}$ with respect to an involutive fuzzy negation $N$ if Equations 39 and 40 holds for all $x, y \in[0,1]$. Let us for example focus on the standard negation $N_{S}$. The operators $C_{M}$ and $I_{K D}$ as well as the operators $C_{L}$ and $I_{L}$ are dual with respect to $N_{S}$.

The concept of adjunction yields another concept of duality which does not require an involutive fuzzy negation. We say that a fuzzy conjunction $C_{\mathcal{F}}$ and a fuzzy implication $I_{\mathcal{F}}$ form an adjunction if for every $a \in[0,1]$, the pair $\left(I_{\mathcal{F}}(a, \cdot), C_{\mathcal{F}}(a, \cdot)\right)$ forms an adjunction on $[0,1]$ in the sense of Equation 4. For example, the pairs $\left(C_{M}, I_{G}\right)$, $\left(C_{L}, I_{L}\right)$, and $\left(C_{K D}, I_{K D}\right)$ represent adjunctions. Therefore, Proposition 1 implies that $I_{\mathcal{F}}(a, \cdot)$ is an erosion and $C_{\mathcal{F}}(a, \cdot)$ is a dilation on $[0,1]$. In other words, Equations 41 and 42 hold true for every every $a, x_{j} \in[0,1]$ and for every set of indices $J$.

$$
\begin{aligned}
I_{\mathcal{F}}\left(a, \bigwedge_{j \in J} x_{j}\right) & =\bigwedge_{j \in J} I_{\mathcal{F}}\left(a, x_{j}\right), \\
C_{\mathcal{F}}\left(a, \bigvee_{j \in J} x_{j}\right) & =\bigvee_{j \in J} C_{\mathcal{F}}\left(a, x_{j}\right) .
\end{aligned}
$$

Equations 41 and 42 will turn out to be useful for the construction of fuzzy erosions and dilations.

\subsection{Fuzzy Mathematical Morphology Based on Fuzzy Inclusion and Intersection Measures}

Recall that in Equation 5 we defined the binary erosion of a set $\mathbf{A}$ by a structuring element $\mathbf{S}$ as the set of all points $\mathbf{x}$ such that the translated structuring element $\mathbf{S}_{\mathbf{x}}$ is contained in A. Formally, we obtain the following equivalent definition of $E_{\mathcal{B}}(\mathbf{A}, \mathbf{S})$.

$$
E_{\mathcal{B}}(\mathbf{A}, \mathbf{S})=\left\{\mathbf{x} \in \mathbf{X}: \operatorname{Inc}\left(\mathbf{S}_{\mathbf{x}}, \mathbf{A}\right)=1\right\}
$$

where Inc : $\mathcal{P}(\mathbf{X}) \times \mathcal{P}(\mathbf{X}) \rightarrow\{0,1\}$ represents the set inclusion for crisp set. We also defined the binary dilation of $\mathbf{A}$ by $\mathbf{S}$ as the set of all $\mathbf{x}$ such that the reflection of $\mathbf{S}_{\mathbf{x}}$ hits $\mathbf{A}$. This notion can be expressed as follows in terms of the intersection $S e c: \mathcal{P}(\mathbf{X}) \times \mathcal{P}(\mathbf{X}) \rightarrow\{0,1\}$ of crisp sets.

$$
D_{\mathcal{B}}(\mathbf{A}, \mathbf{S})=\left\{\mathbf{x} \in \mathbf{X}: \operatorname{Sec}\left(\check{\mathbf{S}}_{\mathbf{x}}, \mathbf{A}\right)=1\right\} .
$$

A consistent fuzzy morphology should be based on definitions of fuzzy erosion and fuzzy dilation that extend the definitions of binary erosion and dilation to the fuzzy domain. This goal can be achieved as follows.

Let a fuzzified set inclusion $I n c_{\mathcal{F}}$ be defined as a $\mathcal{F}(\mathbf{X}) \times$ $\mathcal{F}(\mathbf{X}) \rightarrow[0,1]$ mapping whose restriction to $\mathcal{P}(\mathbf{X}) \times \mathcal{P}(\mathbf{X})$ coincides with the set inclusion for crisp sets. Formally, we have the following implications for all $\mathbf{A}, \mathbf{B} \in \mathcal{P}(\mathbf{X})$ and their corresponding fuzzy sets $\mathbf{a}, \mathbf{b} \in \mathcal{F}(\mathbf{X})$.

$$
\begin{gathered}
\mathbf{A} \subseteq \mathbf{B} \Rightarrow \operatorname{In} c_{\mathcal{F}}(\mathbf{a}, \mathbf{b})=\operatorname{Inc}(\mathbf{A}, \mathbf{B})=1 \\
\mathbf{A} \nsubseteq \mathbf{B} \Rightarrow \operatorname{In} c_{\mathcal{F}}(\mathbf{a}, \mathbf{b})=\operatorname{Inc}(\mathbf{A}, \mathbf{B})=0 .
\end{gathered}
$$

The value $\operatorname{Inc} c_{\mathcal{F}}(\mathbf{a}, \mathbf{b})$ is interpreted as the degree of subsethood or inclusion of the fuzzy set $\mathbf{a}$ in the fuzzy set $\mathbf{b}$.

Various researchers have set out to define fuzzy inclusion measures. Among these definitions are the inclusion measures of Zadeh, Sinha and Dougherty, Kitainik, and Bandler and Kohout. Straightforward verification shows that all of these concepts fuzzify the notion of crisp set inclusion, i.e. they satisfy Equations 45 and 46.

A fuzzy operation $E_{\mathcal{F}}: \mathcal{F}(\mathbf{X}) \times \mathcal{F}(\mathbf{X}) \rightarrow \mathcal{F}(\mathbf{X})$ based on a certain fuzzy inclusion measure $I n c_{\mathcal{F}}$ arises via the following definition [13]:

$$
E_{\mathcal{F}}(\mathbf{a}, \mathbf{s})(\mathbf{x})=\operatorname{Inc} c_{\mathcal{F}}\left(\mathbf{s}_{\mathbf{x}}, \mathbf{a}\right)
$$

where $\mathbf{s}_{\mathbf{x}}(\mathbf{y})$ denotes the translation of $\mathbf{s}$ by $\mathbf{x}$, i.e., $\mathbf{s}_{\mathbf{x}}(\mathbf{y})=$ $\mathbf{s}(\mathbf{y}-\mathbf{x})$, for every $\mathbf{x}, \mathbf{y} \in \mathbf{X}$. Note that $E_{\mathcal{F}}$ extends the binary erosion $E_{\mathcal{B}}: \mathcal{P}(\mathbf{X}) \times \mathcal{P}(\mathbf{X}) \rightarrow \mathcal{P}(\mathbf{X})$ to the fuzzy domain.

We would like to clarify a fact that has not been mentioned in Nachtegael's and Kerre's influential article [13]. We may only refer to $E_{\mathcal{F}}$ as a fuzzy erosion if the operators $\operatorname{Inc} c_{\mathcal{F}}(\mathbf{s},$.$) commute with the infimum operation for all$ 
$\mathbf{s} \in \mathcal{F}(\mathbf{X})$. Otherwise, the operator $E_{\mathcal{F}}(., \mathbf{s})$ does not represent an erosion by the structuring element s since Equation 2 is not satisfied.

In analogy to the measure $I n c_{\mathcal{F}}$, we define a fuzzified set intersection $S e c_{\mathcal{F}}$ defined as a $\mathcal{F}(\mathbf{X}) \times \mathcal{F}(\mathbf{X}) \rightarrow[0,1]$ mapping whose restriction to $\mathcal{P}(\mathbf{X}) \times \mathcal{P}(\mathbf{X})$ coincides with the set intersection for crisp sets. We interpret the value $\operatorname{Sec}_{\mathcal{F}}(\mathbf{a}, \mathbf{b})$ as the degree of intersection of the fuzzy sets $\mathbf{a}$ and $\mathbf{b}$ or the degree of the fuzzy set $\mathbf{a}$ hitting the fuzzy set $\mathbf{b}$. Given a fuzzified set intersection $S e c_{\mathcal{F}}$ such that $\operatorname{Sec}_{\mathcal{F}}(\mathbf{s},$. commutes with the supremum operation, we obtain a function $D_{\mathcal{F}}: \mathcal{F}(\mathbf{X}) \times \mathcal{F}(\mathbf{X}) \rightarrow \mathcal{F}(\mathbf{X})$ by setting

$$
D_{\mathcal{F}}(\mathbf{a}, \mathbf{s})(\mathbf{x})=\operatorname{Sec}\left(\check{\mathbf{s}}_{\mathbf{x}}, \mathbf{a}\right),
$$

where š denotes the reflection around the origin, i.e., $\check{\mathbf{s}}(\mathbf{x})=$ $\mathbf{s}(-\mathbf{x})$, for every $\mathbf{x} \in \mathbf{X}$. Note that $D_{\mathcal{F}}$ coincides with $D_{\mathcal{B}}$ on $\mathcal{P}(\mathbf{X}) \times \mathcal{P}(\mathbf{X})$. We refer to $D_{\mathcal{F}}$ using the terminology fuzzy dilation if the operator $D_{\mathcal{F}}(\cdot, \mathbf{s})$ represents a dilation for every $\mathbf{s} \in \mathcal{F}(\mathbf{X})$ in the sense of Equation 3, i.e. if $D(\cdot, \mathbf{s})$ commutes with the supremum operation for every $\mathbf{s} \in \mathcal{F}(\mathbf{X})$.

\subsection{Fuzzy Inf-I Inclusion and the Sup-C Intersec- tion Measures}

This section explains how a fuzzy inclusion measure can be derived from the crisp inclusion measure.

Let $I:\{0,1\} \times\{0,1\} \rightarrow\{0,1\}$ denote the crisp implication. Consider arbitrary crisp sets $\mathbf{A}, \mathbf{B} \subseteq \mathbf{X}$. Obviously, we have $\mathbf{A} \subseteq \mathbf{B}$ if and only if $\mathbf{x} \in \mathbf{A}$ implies that $\mathbf{x} \in \mathbf{B}$ for all $\mathbf{x} \in \mathbf{X}$. This statement can be reformulated as follows:

$$
\operatorname{Inc}(\mathbf{A}, \mathbf{B})=\bigwedge_{\mathbf{x} \in \mathbf{X}} I(\mathbf{A}(\mathbf{x}), \mathbf{B}(\mathbf{x})) .
$$

Now consider arbitrary fuzzy sets $\mathbf{a}, \mathbf{b} \in \mathcal{F}(\mathbf{X})$. A straightforward fuzzification of Equation 49 leads to the following fuzzy inclusion measure $I n c_{\mathcal{F}}$ [2]:

$$
\operatorname{Inc_{\mathcal {F}}}(\mathbf{a}, \mathbf{b})=\bigwedge_{\mathbf{x} \in \mathbf{X}} I_{\mathcal{F}}(\mathbf{a}(\mathbf{x}), \mathbf{b}(\mathbf{x})) .
$$

A fuzzy operation $I n c_{\mathcal{F}}$ of this form will be called fuzzy Inf-I inclusion measure. Clearly, the restriction of $I n c_{\mathcal{F}}$ to $\mathcal{P}(\mathbf{X}) \times \mathcal{P}(\mathbf{X})$ is given by the crisp set inclusion Inc since $I$ represents the restriction of $I_{\mathcal{F}}$ to $\mathcal{P}(\mathbf{X})$.

Following a similar line of reasoning, we derive a fuzzy intersection measure $S e c_{\mathcal{F}}$ by means of the following equation.

$$
\operatorname{Sec}_{\mathcal{F}}(\mathbf{a}, \mathbf{b})=\bigvee_{\mathbf{x} \in \mathbf{X}} C_{\mathcal{F}}(\mathbf{a}(\mathbf{x}), \mathbf{b}(\mathbf{x})) .
$$

We will call a fuzzy operation $S e c_{\mathcal{F}}$ of this form fuzzy Sup-C intersection measure. This operation $S e c_{\mathcal{F}}$ fuzzifies the crisp set intersection measure.
Now let us reconsider the operation $E_{\mathcal{F}}: \mathcal{F}(\mathbf{X}) \times$ $\mathcal{F}(\mathbf{X}) \rightarrow \mathcal{F}(\mathbf{X})$ described in Equation 47. Let us assume that $I n c_{\mathcal{F}}$ is given by a Inf-I inclusion measure. Recall that the operators $E_{\mathcal{F}}(\cdot, \mathbf{s}): \mathcal{F}(\mathbf{X}) \rightarrow \mathcal{F}(\mathbf{X})$ represent erosions for all $\mathbf{s} \in \mathcal{F}(\mathbf{X})$ if and only if $\operatorname{Inc_{\mathcal {F}}}(\mathbf{s}, \cdot)$ commute with the infimum operation for all $\mathbf{s} \in \mathcal{F}(\mathbf{X})$. The latter statement is certainly true if we have for all $s \in[0,1]$ that the operators $I_{\mathcal{F}}(s, \cdot)$ commute with the infimum, in other words if $I_{\mathcal{F}}(s, \cdot)$ are erosions in $[0,1]$. In this case, we have for all index sets $J$ and for all $\mathbf{s}, \mathbf{a}_{j} \in \mathcal{F}(\mathbf{X})$ :

$$
\begin{array}{r}
\quad \operatorname{Inc}_{\mathcal{F}}\left(\mathbf{s}, \bigwedge_{j \in J} \mathbf{a}_{j}\right)=\bigwedge_{\mathbf{x} \in \mathbf{X}} I_{\mathcal{F}}\left(\mathbf{s}(\mathbf{x}), \bigwedge_{j \in J} \mathbf{a}_{j}(\mathbf{x})\right) \\
=\bigwedge_{\mathbf{x} \in \mathbf{X}}\left[\bigwedge_{j \in J} I_{\mathcal{F}}\left(\mathbf{s}(\mathbf{x}), \mathbf{a}_{j}(\mathbf{x})\right)\right]=\bigwedge_{j \in J} \operatorname{Inc} c_{\mathcal{F}}\left(\mathbf{s}, \mathbf{a}_{j}\right),
\end{array}
$$

For example, the implications $I_{G}, I_{L}$, and $I_{K D}$ commute with the infimum operation in the second argument and thus Equation 47 yields an erosion by the structuring element $\mathrm{s}$ if $I n c_{\mathcal{F}}$ is given in terms of one of the corresponding Inf-I inclusion measures. However, the Inf-I inclusion measure does not yield a fuzzy erosion in terms of Equation 47 for the following fuzzy implication $I_{C E}$.

$$
I_{C E}(x, y)= \begin{cases}0, & \text { if } x=1 \text { and } y=0 \\ 1, & \text { otherwise }\end{cases}
$$

Similar observations can be made for the situation where the fuzzified set intersection of Equation 48 is given by a Sup-C intersection measure. If $C_{\mathcal{F}}(s, \cdot)$ is a dilation in $[0,1]$ for every $s \in[0,1]$ then the operator $D_{\mathcal{F}}(\cdot, \mathbf{s})$ is a dilation for every $\mathbf{s} \in \mathcal{F}(\mathbf{X})$. In this case, we speak of the fuzzy dilation of the image a by the structuring element $\mathbf{s}$.

To our knowledge, almost all approaches towards fuzzy mathematical morphology are based on fuzzy erosions as defined in Equation 47 and fuzzy dilations as defined in Equation 48 Moreover, the inclusion measure appearing in Equation 47 is determined by an infimum of implications and the intersection measure appearing in Equation 47 is determined by a supremum of conjunctions. In this case, we can rewrite Equations 47 and 48 as follows.

$$
\begin{aligned}
E_{\mathcal{F}}(\mathbf{a}, \mathbf{s})(\mathbf{x}) & =\bigwedge_{\mathbf{y} \in \mathbf{X}} I_{\mathcal{F}}\left(\mathbf{s}_{\mathbf{x}}(\mathbf{y}), \mathbf{a}(\mathbf{y})\right), \\
D_{\mathcal{F}}(\mathbf{a}, \mathbf{s})(\mathbf{x}) & =\bigvee_{\mathbf{y} \in \mathbf{X}} C_{\mathcal{F}}\left(\check{\mathbf{s}}_{\mathbf{x}}(\mathbf{y}), \mathbf{a}(\mathbf{y})\right) .
\end{aligned}
$$

Equivalently, we have

$$
\begin{aligned}
E_{\mathcal{F}}(\mathbf{a}, \mathbf{s})(\mathbf{x}) & =\bigwedge_{\mathbf{y} \in \mathbf{X}} I_{\mathcal{F}}(\mathbf{s}(\mathbf{y}-\mathbf{x}), \mathbf{a}(\mathbf{y})), \\
D_{\mathcal{F}}(\mathbf{a}, \mathbf{s})(\mathbf{x}) & =\bigvee_{\mathbf{y} \in \mathbf{X}} C_{\mathcal{F}}(\mathbf{s}(\mathbf{x}-\mathbf{y}), \mathbf{a}(\mathbf{y})) .
\end{aligned}
$$


De Baets allows for an arbitrary implication in Equation 57 and for an arbitrary conjunction in Equation 58. Other researchers impose the following restrictions. Some approaches are restricted to specific choices of $I_{\mathcal{F}}$ and $C_{\mathcal{F}}$. Some researchers require that the conjunction $C_{\mathcal{F}}$ in Equation 58 is the dual of the implication $I_{\mathcal{F}}$ in Equation 57 with respect to a (particular) involutive negation. Other researchers require that $C_{\mathcal{F}}$ and $I_{\mathcal{F}}$ are adjoint.

From now on, subscripts of the symbol $E_{\mathcal{F}}$ and $D_{\mathcal{F}}$ will indicate the type of implications that is used in Equations 57 and 58. For example, the symbol $E_{G}$ stands for the fuzzy erosion that is given by the equation $E_{G}(\mathbf{a}, \mathbf{s})(\mathbf{x})=$ $\bigwedge_{\mathbf{y} \in \mathbf{X}} I_{G}(\mathbf{s}(\mathbf{y}-\mathbf{x}), \mathbf{a}(\mathbf{y}))$. The following theorems exhibit the connections between the fuzzy erosion defined in Equation 57, the fuzzy dilation defined in Equation 58 respectively, and the L-erosion and U-erosion, the L-dilation and the U-dilation respectively. The proofs are straightforward.

Theorem 6. The following equations hold for all $\mathbf{a}, \mathbf{s} \in$ $\mathcal{F}(\mathbf{X})$ and for all $\mathbf{x} \in \mathbf{X}$.

$$
\begin{array}{r}
E_{G}(\mathbf{a}, \mathbf{s})(\mathbf{x})=E_{\mathcal{L}}(\mathbf{a}, \mathbf{s})(\mathbf{x}) \wedge 1, \\
D_{M}(\mathbf{a}, \mathbf{s})(\mathbf{x})=D_{\mathcal{L}}(\mathbf{a}, \mathbf{s})(\mathbf{x}), \\
E_{L}(\mathbf{a}, \mathbf{s})(\mathbf{x})=\left[E_{\mathcal{U}}(\mathbf{a}, \mathbf{s})(\mathbf{x})+1\right] \wedge 1, \\
D_{L}(\mathbf{a}, \mathbf{s})(\mathbf{x})=\left[D_{\mathcal{U}}(\mathbf{a}, \mathbf{s})(\mathbf{x})-1\right] \vee 0 .
\end{array}
$$

Example 6. Let a $\in \mathcal{F}(\mathbf{X})$ be the fuzzy image and let $\mathbf{s} \in \mathcal{F}(\mathbf{X})$ be the structuring element given by

$$
\mathbf{s}(\mathbf{x})=((1+\mathbf{x}) \vee 0) \wedge((1-\mathbf{x}) \vee 0) .
$$

Figure 5 displays the results of applications of the fuzzy erosions $E_{G}, E_{L}$, and $E_{K D}$. The dashed line represents the original image a. The pointed line represents the outputs of fuzzy erosion $E_{L}$. The point-dashed line and the continuous line represent the fuzzy erosion $E_{G}(\mathbf{a}, \mathbf{s})$ and $E_{K D}(\mathbf{a}, \mathbf{s})$, respectively.

\section{References}

[1] B. D. Baets, E. Kerre, and M. Gupta. The fundamentals of fuzzy mathematical morphology, part 1: basic concepts. International Journal of General Systems, 23:155-171, 1994.

[2] W. Bandler and L. Kohout. Fuzzy power sets and fuzzy implication operators. Fuzzy Sets and Systems, 4(1):13-30, July 1980 .

[3] G. Banon. Characterization of translation-invariant elementary morphological operators for gray-level morphology. In Neural, Morphological, and Stochastic Methods in Image and Signal Processing, volume 2568 of Proceedings of SPIE, pages 68-79, San Diego, CA, July 1995.

[4] G. J. F. Banon. Characterization of translation-invariant elementary morphological operators between gray-level

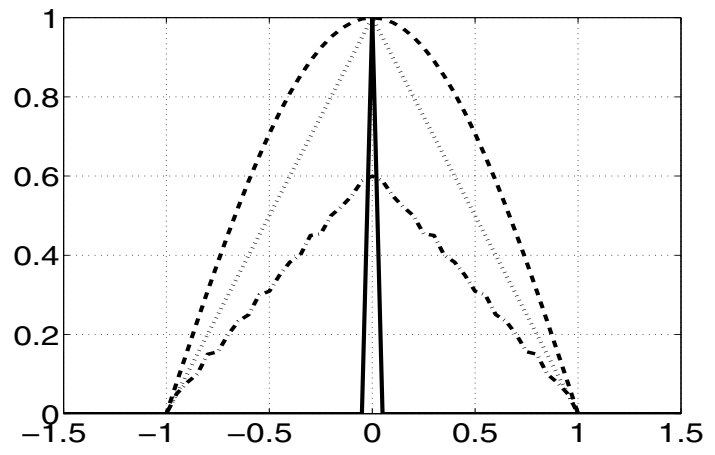

Figure 5. The images $E_{L}(\mathbf{a}, \mathbf{s}), E_{G}(\mathbf{a}, \mathbf{s})$, and $E_{K D}(\mathbf{a}, \mathbf{s})$ correspond to the pointed line, the pointdashed line, and the continuous line.

images. Technical report, INPE, São José dos Campos, 1995. Avaliable at: http://iris.sid.inpe.br:1912/rep/dpi.inpe.br/banon/1995/10.20.17.45.

[5] G. Birkhoff. Lattice Theory. American Mathematical Society, Providence, 3 edition, 1993.

[6] I. Bloch and H. Maitre. Fuzzy mathematical morphologies: acomparative study. Pattern Recognition, 28(9):1341-1387, 1995.

[7] T. Deng and H. Heijmans. Grey-scale morphology based on fuzzy logic. Journal of Mathematical Imaging and Vision, 16(2):155-171, Mar. 2002.

[8] H. Hadwiger. Vorlesungen Über Inhalt, Oberfläche und Isoperimetrie. Springer-Verlag, Berlin, 1957.

[9] H. Heijmans. Morphological Image Operators. Academic Press, New York, NY, 1994.

[10] L. Kitainik. Fuzzy Decision Procedures with Binary Relations. Kluwer Academic Publishers, 1993.

[11] G. Matheron. Random Sets and Integral Geometry. Wiley, New York, 1975.

[12] H. Minkowski. Gesammelte Abhandlungen. Teubner Verlag, Leipzig-Berlin, 1911.

[13] M. Nachtegael and E. Kerre. Connections between binary, gray-scale and fuzzy mathematical morphologies. Fuzzy Sets and Systems, 124(1):73-85, Nov. 2001.

[14] C. Ronse. Why mathematical morphology needs complete lattices. Signal Processing, 21(2):129-154, Oct. 1990.

[15] J. Serra. Image Analysis and Mathematical Morphology. Academic Press, London, 1982.

[16] J. Serra. Image Analysis and Mathematical Morphology, Volume 2: Theoretical Advances. Academic Press, New York, 1988.

[17] S. Sinha and E. R. Dougherty. A general axiomatic theory of intrinsically fuzzy mathematical morphologies. IEEE Transactions on Fuzzy Systems, 3(4):389-403, November 1995.

[18] S. Sternberg. Parallel architecture for image processing. In Proceedings of the Third International IEEE Compsac, Chicago, 1979.

[19] S. Sternberg. Biomedical image processing. Computer, 16(1), Jan. 1983.

[20] L. A. Zadeh. Fuzzy sets. Information and Control, 8(3):338353, 1965. 